\title{
Progress in the multidisciplinary management of esophageal cancer: what are the latest developments?
}

\author{
Thomas Winder
}

Received: 18 January 2019 / Accepted: 29 January 2019

(C) Springer-Verlag GmbH Austria, part of Springer Nature 2019

In the last decade strategies to improve overall survival of patients with esophageal cancer have changed. To summarize this dynamic development the topic for the authors of this mini-review series of esophageal cancer was to highlight the latest developments in the multidisciplinary treatment of patients with esophageal cancer.

Despite living in a dynamic field of personalized medicine using the molecular make-up of each individual tumor for targeted treatment strategies or to release the breaks of the immune system by checkpoint inhibitors, there are indeed also dramatic improvements in screening procedures of Barrett esophagus, local treatments such as surgery or radiotherapy and evolving minimally invasive treatment strategies.

Globally, esophageal cancer is the ninth most common cancer and the sixth most common cause of cancer-related death. Although incidence of squamous cell carcinoma of the esophagus is decreasing in several areas, a significant increase in adenocarcinoma has been noted in several regions such as Europe. The 5 -year overall survival is about $20 \%$ and strongly dependent on several factors such as tumor stage, tumor location, molecular markers, patient's individual factors such reflux, obesity, alcohol and cigarette smoking, as well as performance status and comorbidities. In addition, hospital and surgeon volume on esophagectomies are key for clinical outcome. Highvolume centers have a lower mortality rate than lowvolume centers. Therefore, an environment with an

PD Dr. T. Winder, PhD ( $\bowtie)$

Department of Internal Medicine II, Medical Oncology, Hematology and Gastroenterology, Academic Teaching Hospital Feldkirch, Carinagasse 47, 6807 Feldkirch, Austria thomas.winder@vlkh.net

Swiss Tumor Molecular Institute, Zürich, Switzerland experienced multidisciplinary team is of upmost importance to further improve overall survival of patients with esophageal cancer.

Over the past decade, there have been practicechanging developments in the treatment of patients with esophageal cancer. Endoscopic screening and ablative procedures are key in the identification of Barrett's esophagus and efficacious for the treatment of dysplasia and early esophageal cancer. Radiotherapy has an ongoing development by using cutting edge techniques. Neoadjuvant combined radiochemotherapy is standard prior to surgery for resectable, locally advanced esophageal cancer. Molecular pathology divides esophageal cancer into different subtypes, enabling in the future more individualized treatment strategies such as immunotherapy or targeted treatment and identifying novel targets. Surgery has become more standardized and centralized and continues to develop minimally invasive strategies such as robotic-assisted minimally invasive esophagectomy to further improve the outcome of patients with esophageal cancer.

Multidisciplinary management in well-experienced centers will remain the mainstay in the treatment of esophageal cancer. The major issues will be to increase the detection of premalignant lesions and early stage tumors, new diagnostic measures to improve specificity and sensitivity for a more accurate assessment of disease stage and finally, further developed systemic and local treatments are required for the next breakthrough in the prognosis of esophageal cancer.

The authors of this mini-review series should be congratulated for this comprehensive and precise presentation of the latest developments in esophageal cancer.

Conflict of interest T. Winder declares that he has no competing interests. 
Publisher's Note Springer Nature remains neutral with regard to jurisdictional claims in published maps and institutional affiliations.

For latest news from international oncology congresses see: http://www.springermedizin.at/ memo-inoncology 